\title{
A Class of Estimators of Population Mean in Case of Post Stratification
}

Hilal A. Lone

School of Studies in Statistics

Vikram University, Ujjain-456010, M.P. India

hilalstat@gmail.com

Rajesh Tailor

School of Studies in Statistics,

Vikram University, Ujjain-456010, M.P. India

tailorraj@gmail.com

\begin{abstract}
This paper proposes a class of ratio-cum-product type estimators in case of post-stratification. Particular members of the proposed class of ratio-cum-product type estimators have been identified and studied thoroughly from efficiency point of view. It has been shown that the identified particular estimators are more efficient than the usual unbiased estimator, Ige and Tripathi (1989) estimators, Chouhan (2012) estimators, Tailor et al. (2016) estimator and other considered estimators. An empirical study has been carried out to demonstrate the performance of the proposed estimators.
\end{abstract}

Keywords: Mean squared error, Bias, Ratio-cum-product estimator.

\section{Introduction}

Cochran (1940) and Robson (1957) envisaged classical ratio and product estimators which were studied in case of post stratification by Ige and Tripathi (1989). Recently, Lone and Tailor (2014) and Lone and Tailor (2015) proposed ratio and product type exponential estimators in case of post-stratification. Chouhan (2012) proposed class of ratio type estimators using various known parameters of auxiliary variates in case of post stratification. Tailor et al. (2015) proposed dual to Ige and Tripathi (1989) ratio and product estimators. Tailor et al. (2016) proposed a ratio-cum-product type estimator in case of post-stratification. Singh (1967) used information on population mean of two auxiliary variates and proposed ratio-cum-product type estimator for population mean in simple random sampling. Singh (1967) and Chouhan (2012) motivate authors to propose the class of ratio-cum-product type estimators in case of post-stratification. Many Researchers including Holt and Smith (1979), Jagers et al. (1985), Jagers (1986), Ige and Tripathi (1989), Agrawal and Panday (1993), Singh and Ruiz Espejo (2003) Tailor et al. (2011) and Tailor et al. (2015) contributed well in case of post-stratification.

Let us consider a finite population $U=\left(U_{1}, U_{2}, \ldots, U_{N}\right)$ of size $N$ which is divided into L strata of size $N_{1}, N_{2}, \ldots, N_{L}$ such that $\sum_{h=1}^{L} N_{h}=N$. Let $y$ be the study variate and $x$ is the auxiliary variate correlated positively with the study variate $y$ and $z$ is the auxiliary 
variate, negatively correlated with the study variate $y$. Let $y_{h i}$ be the observation on $i^{\text {th }}$ unit of $h^{\text {th }}$ stratum for study variate $y$ and $x_{h i}$ and $z_{h i}$ be the observation on $i^{\text {th }}$ unit of $h^{\text {th }}$ stratum for auxiliary variates $x$ and $z$ respectively, then

$\bar{X}_{h}=\frac{1}{N_{h}} \sum_{i=1}^{N_{h}} x_{h i}: h^{t h}$ stratum mean for the auxiliary variate $x$,

$\bar{Y}_{h}=\frac{1}{N_{h}} \sum_{i=1}^{N_{h}} y_{h i}: h^{t h}$ stratum mean for the study variate $y$,

$\bar{Z}_{h}=\frac{1}{N_{h}} \sum_{i=1}^{N_{h}} x_{h i}: h^{\text {th }}$ stratum mean for the auxiliary variate $z$,

$\bar{X}=\frac{1}{N} \sum_{h=1}^{L} \sum_{i=1}^{N_{h}} x_{h i}=\sum_{h=1}^{L} W_{h} \bar{X}_{h}:$ Population mean of the auxiliary variate $x$,

$\bar{Y}=\frac{1}{N} \sum_{h=1}^{L} \sum_{i=1}^{N_{h}} y_{h i}=\sum_{h=1}^{L} W_{h} \bar{Y}_{h}:$ Population mean of the study variate $y$ and

$\bar{Z}=\sum_{h=1}^{L} W_{h} \bar{Z}_{h}$ : Population mean of the auxiliary variate $z$.

A sample of size $n$ is drawn from population $U$ using simple random sampling without replacement. After selecting the sample, it is observed that which units belong to $h^{\text {th }}$ stratum. Let $n_{h}$ be the size of the sample falling in $h^{\text {th }}$ stratum such that $\sum_{h=1}^{L} n_{h}=n$ Here it is assumed that $n$ is so large that possibility of $n_{h}$ being zero is very small.

In case of post-stratification, usual unbiased estimator of population mean $\bar{Y}$ is defined as

$$
\bar{y}_{P S}=\sum_{h=1}^{L} W_{h} \bar{y}_{h}
$$

where

$W_{h}=\frac{N_{h}}{N}$ is the weight of the $h^{\text {th }}$ stratum and $\bar{y}_{h}=\frac{1}{n_{h}} \sum_{i=1}^{n_{h}} y_{h i}$ is sample mean of $n_{h}$ sample units that fall in the $h^{\text {th }}$ stratum.

Using the results from Stephen (1945), the variance of $\bar{y}_{P S}$ to the first degree of approximation is obtained as

$$
\operatorname{Var}\left(\bar{y}_{P S}\right)=\left(\frac{1}{n}-\frac{1}{N}\right) \sum_{h=1}^{L} W_{h} S_{y h}^{2}+\frac{1}{n^{2}} \sum_{h=1}^{L}\left(1-W_{h}\right) S_{y h}^{2}
$$


where $S_{y h}^{2}=\frac{1}{N_{h}-1} \sum_{i=1}^{N_{h}}\left(y_{h i}-\bar{Y}_{h}\right)^{2}$.

Ige and Tripathi (1989) defined a ratio and a product type estimator in case of poststratification as

$$
\hat{\bar{Y}}_{P S}^{R}=\bar{y}_{P S}\left(\frac{\bar{X}}{\bar{x}_{P S}}\right)
$$

and

$$
\hat{\bar{Y}}_{P S}^{P}=\bar{y}_{P S}\left(\frac{\bar{z}_{P S}}{\bar{Z}}\right)
$$

where $\bar{x}_{P S}=\sum_{h=1}^{L} W_{h} \bar{x}_{h}$ and $\bar{z}_{P S}=\sum_{h=1}^{L} W_{h} \bar{z}_{h}$ are the unbiased estimators of population means in case of post-stratification and $\bar{x}_{h}$ and $\bar{z}_{h}$ are the means of the samples of size $n_{h}$ that in case of post-stratification and $\bar{x}_{h}$ and $\bar{z}_{h}$ are the means of the samples of size fall in $h^{\text {th }}$ stratum.

Mean squared error of the Ige and Tripathi (1989) estimators $\hat{\bar{Y}}_{P S}^{R}$ and $\hat{\bar{Y}}_{P S}^{P}$ are

$$
\operatorname{MSE}\left(\hat{\bar{Y}}_{P S}^{R}\right)=\left(\frac{1}{n}-\frac{1}{N}\right) \sum_{h=1}^{L} W_{h}\left(S_{y h}^{2}+R_{1}^{2} S_{x h}^{2}-2 R_{1} S_{y x h}\right)
$$

and

$$
\operatorname{MSE}\left(\hat{\bar{Y}}_{P S}^{P}\right)=\left(\frac{1}{n}-\frac{1}{N}\right) \sum_{h=1}^{L} W_{h}\left(S_{y h}^{2}+R_{2}^{2} S_{z h}^{2}+2 R_{2} S_{y z h}\right) .
$$

where

$$
R_{1}=\frac{\bar{Y}}{\bar{X}} \quad \text { and } \quad R_{2}=\frac{\bar{Y}}{\bar{Z}} .
$$

Chouhan (2012) proposed the following ratio type estimators for population mean $\bar{Y}$ in case of post-stratification as

$$
\begin{aligned}
& \hat{\bar{Y}}_{P S}^{R S D}=\bar{y}_{p s}\left(\frac{\sum_{h=1}^{L} W_{h}\left(\bar{X}_{h}+C_{x h}\right)}{\sum_{h=1}^{L} W_{h}\left(\bar{x}_{h}+C_{x h}\right)}\right), \\
& \hat{\bar{Y}}_{P S}^{R S E}=\bar{y}_{p s}\left(\frac{\sum_{h=1}^{L} W_{h}\left(\bar{X}_{h}+\beta_{2 h}(x)\right)}{\sum_{h=1}^{L} W_{h}\left(\bar{x}_{h}+\beta_{2 h}(x)\right)}\right)
\end{aligned}
$$

and 


$$
\hat{\bar{Y}}_{P S}^{R S T}=\bar{y}_{p s}\left(\frac{\sum_{h=1}^{L} W_{h}\left(\bar{X}_{h}+\rho_{y x h}\right)}{\sum_{h=1}^{L} W_{h}\left(\bar{x}_{h}+\rho_{y x h}\right)}\right),
$$

Mean squared errors of the estimators $\hat{\bar{Y}}_{P S}^{R S D}, \hat{\bar{Y}}_{P S}^{R S E}$ and $\hat{\bar{Y}}_{P S}^{R S T}$ upto the first degree of approximation are obtained as

$$
\begin{aligned}
& \operatorname{MSE}\left(\hat{\bar{Y}}_{P S}^{R S D}\right)=\left(\frac{1}{n}-\frac{1}{N}\right) \sum_{h=1}^{L} W_{h}\left(S_{y h}^{2}+R_{n 1}^{2} S_{x h}^{2}-2 R_{n 1} S_{y x h}\right), \\
& \operatorname{MSE}\left(\hat{\bar{Y}}_{P S}^{R S E}\right)=\left(\frac{1}{n}-\frac{1}{N}\right) \sum_{h=1}^{L} W_{h}\left(S_{y h}^{2}+R_{n 2}^{2} S_{x h}^{2}-2 R_{n 2} S_{y x h}\right), \\
& \operatorname{MSE}\left(\hat{\bar{Y}}_{P S}^{R S T}\right)=\left(\frac{1}{n}-\frac{1}{N}\right) \sum_{h=1}^{L} W_{h}\left(S_{y h}^{2}+R_{n 3}^{2} S_{x h}^{2}-2 R_{n 3} S_{y x h}\right),
\end{aligned}
$$

Where as the product version of $\hat{\bar{Y}}_{P S}^{R S D}, \hat{\bar{Y}}_{P S}^{R S E}$ and $\hat{\bar{Y}}_{P S}^{R S T}$ can be written as

$$
\begin{gathered}
\hat{\bar{Y}}_{P S}^{P S D}=\bar{y}_{p s}\left(\frac{\sum_{h=1}^{L} W_{h}\left(\bar{z}_{h}+C_{z h}\right)}{\sum_{h=1}^{L} W_{h}\left(\bar{Z}_{h}+C_{z h}\right)}\right), \\
\hat{\bar{Y}}_{P S E}^{P S}=\bar{y}_{p s}\left(\frac{\sum_{h=1}^{L} W_{h}\left(\bar{z}_{h}+\beta_{2 h}(z)\right)}{\sum_{h=1}^{L} W_{h}\left(\bar{Z}_{h}+\beta_{2 h}(z)\right)}\right)
\end{gathered}
$$

and

$$
\hat{\bar{Y}}_{P S}^{P S T}=\bar{y}_{p s}\left(\frac{\sum_{h=1}^{L} W_{h}\left(\bar{z}_{h}+\rho_{y z h}\right)}{\sum_{h=1}^{L} W_{h}\left(\bar{Z}_{h}+\rho_{y z h}\right)}\right)
$$

Upto the first degree of approximation, mean squared error of the estimators $\hat{\bar{Y}}_{P S}^{P S D}, \hat{\bar{Y}}_{P S}^{P S E}$ and $\hat{\bar{Y}}_{P S}^{P S T}$ are obtained as

$$
\begin{aligned}
& \operatorname{MSE}\left(\hat{\bar{Y}}_{P S}^{P S D}\right)=\left(\frac{1}{n}-\frac{1}{N}\right) \sum_{h=1}^{L} W_{h}\left(S_{y h}^{2}+R_{m 1}^{2} S_{z h}^{2}+2 R_{m 1} S_{y z h}\right), \\
& \operatorname{MSE}\left(\hat{\bar{Y}}_{P S}^{P S E}\right)=\left(\frac{1}{n}-\frac{1}{N}\right) \sum_{h=1}^{L} W_{h}\left(S_{y h}^{2}+R_{m 2}^{2} S_{z h}^{2}+2 R_{m 2} S_{y z h}\right),
\end{aligned}
$$




$$
\operatorname{MSE}\left(\hat{\bar{Y}}_{P S}^{P S T}\right)=\left(\frac{1}{n}-\frac{1}{N}\right) \sum_{h=1}^{L} W_{h}\left(S_{y h}^{2}+R_{m 3}^{2} S_{z h}^{2}+2 R_{m 3} S_{y z h}\right),
$$

where

$$
R_{n i}=\left\{\begin{array}{ll}
\bar{Y} / \sum_{h=1}^{L} W_{h}\left(\bar{X}_{h}+C_{x h}\right) & i=1 \\
\bar{Y} / \sum_{h=1}^{L} W_{h}\left(\bar{X}_{h}+\beta_{2 h}(x)\right) & i=2 \\
\bar{Y} / \sum_{h=1}^{L} W_{h}\left(\bar{X}_{h}+\rho_{y x h}\right) & i=3
\end{array} \quad \text { and } R_{m i}= \begin{cases}\bar{Y} / \sum_{h=1}^{L} W_{h}\left(\bar{Z}_{h}+C_{x h}\right) & i=1 \\
\bar{Y} / \sum_{h=1}^{L} W_{h}\left(\bar{Z}_{h}+\beta_{2 h}(z)\right) & i=2 . \\
\bar{Y} / \sum_{h=1}^{L} W_{h}\left(\bar{Z}_{h}+\rho_{y z h}\right) & i=3\end{cases}\right.
$$

Tailor et al. (2016) proposed a ratio-cum-product type estimator in case of poststratification as

$$
\hat{\bar{Y}}_{P S}^{R P}=\bar{y}_{p s}\left(\frac{\sum_{h=1}^{L} W_{h} \bar{X}_{h}}{\sum_{h=1}^{L} W_{h} \bar{x}_{h}}\right)\left(\frac{\sum_{h=1}^{L} W_{h} \bar{z}_{h}}{\sum_{h=1}^{L} W_{h} \bar{Z}_{h}}\right)
$$

Up to the first degree of approximation, mean squared error of $\hat{\bar{Y}}_{P S}^{R P}$ is obtained as

$$
\operatorname{MSE}\left(\hat{\bar{Y}}_{P S}^{R P}\right)=\left(\frac{1}{n}-\frac{1}{N}\right) \sum_{h=1}^{L} W_{h}\left[S_{y h}^{2}+R_{1}^{2} S_{x h}^{2}+R_{2}^{2} S_{z h}^{2}-2\left(R_{1} S_{y x h}+R_{1} R_{2} S_{x z h}-R_{2} S_{y z h}\right)\right]
$$

\section{Proposed Class of Ratio-Cum- Product Type Estimators}

In the line of Singh (1967), we suggest a class of ratio-cum-product type estimators for estimating population mean $\bar{Y}$ as

$$
t=\bar{y}_{p s}\left(\frac{\sum_{h=1}^{L} W_{h}\left(a_{h} \bar{X}_{h}+b_{h}\right)}{\sum_{h=1}^{L} W_{h}\left(a_{h} \bar{x}_{h}+b_{h}\right)}\right)\left(\frac{\sum_{h=1}^{L} W_{h}\left(c_{h} \bar{z}_{h}+d_{h}\right)}{\sum_{h=1}^{L} W_{h}\left(c_{h} \bar{Z}_{h}+d_{h}\right)}\right)
$$

where $\left(a_{h}, b_{h}, c_{h}\right.$ and $\left.d_{h}\right)$ are the function of population parameters of the auxiliary variates.

To obtain the bias and mean squared error of the proposed estimator $t$, we write

$\bar{y}_{h}=\bar{Y}_{h}\left(1+e_{0 h}\right), \quad \bar{x}_{h}=\bar{X}_{h}\left(1+e_{1 h}\right)$ and $\bar{z}_{h}=\bar{Z}_{h}\left(1+e_{2 h}\right)$ such that

$$
E\left(e_{0 h}\right)=E\left(e_{1 h}\right)=E\left(e_{2 h}\right)=0,
$$




$$
\begin{aligned}
& E\left(e_{0 h}^{2}\right)=\left(\frac{1}{n W_{h}}-\frac{1}{N_{h}}\right) C_{y h}^{2}, \\
& E\left(e_{1 h}^{2}\right)=\left(\frac{1}{n W_{h}}-\frac{1}{N_{h}}\right) C_{x h}^{2}, \\
& E\left(e_{2 h}^{2}\right)=\left(\frac{1}{n W_{h}}-\frac{1}{N_{h}}\right) C_{z h}^{2}, \\
& E\left(e_{0 h} e_{1 h}\right)=\left(\frac{1}{n W_{h}}-\frac{1}{N_{h}}\right) \rho_{y x h} C_{y h} C_{x h}, \\
& E\left(e_{0 h} e_{2 h}\right)=\left(\frac{1}{n W_{h}}-\frac{1}{N_{h}}\right) \rho_{y z h} C_{y h} C_{z h} \text { and } \\
& E\left(e_{1 h} e_{2 h}\right)=\left(\frac{1}{n W_{h}}-\frac{1}{N_{h}}\right) \rho_{x z h} C_{x h} C_{z h} .
\end{aligned}
$$

Expressing (2.1) in terms of e's, we have

$$
\begin{gathered}
t=\bar{Y}\left(1+\frac{\sum_{h=1}^{L} W_{h} \bar{Y}_{h} e_{0 h}}{\bar{Y}}\right)\left(1+\frac{\sum_{h=1}^{L} W_{h} a_{h} \bar{X}_{h} e_{1 h}}{X_{n}}\right)^{-1}\left(1+\frac{\sum_{h=1}^{L} W_{h} c_{h} \bar{Z}_{h} e_{2 h}}{X_{m}}\right) \\
t=\bar{Y}\left(1+e_{0}\right)\left(1+e_{1}\right)^{-1}\left(1+e_{2}\right) \\
\Rightarrow t-\bar{Y}=\bar{Y}\left(e_{0}-e_{1}+e_{2}+e_{1}^{2}-e_{1} e_{2}-e_{0} e_{1}+e_{0} e_{2}\right) \\
\text { where } e_{0}=\frac{\sum_{h=1}^{L} W_{h} \bar{Y}_{h} e_{0 h}}{\bar{Y}}, e_{1}=\frac{\sum_{h=1}^{L} W_{h} a_{h} \bar{X}_{h} e_{1 h}}{X_{n}} \text { and } e_{2}=\frac{\sum_{h=1}^{L} W_{h} c_{h} \bar{Z}_{h} e_{2 h}}{X_{m}} .
\end{gathered}
$$

Taking expectation on both sides of (2.2), we get the bias of the proposed estimator $t$ upto the first degree of approximation as

$$
\begin{aligned}
& B(t)=\left(\frac{1}{n}-\frac{1}{N}\right)\left[\frac{1}{X_{n}}\left\{\sum_{h=1}^{L} W_{h} S_{x h}^{2} a_{h}^{2} R_{n}-\sum_{h=1}^{L} W_{h} a_{h} S_{y x h}\right\}\right. \\
& \left.+\frac{1}{X_{m}}\left\{\sum_{h=1}^{L} W_{h} c_{h} S_{y z h}-\sum_{h=1}^{L} W_{h} R_{n} a_{h} c_{h} S_{x z h}\right\}\right]
\end{aligned}
$$

Squaring and taking expectation on both sides of (2.2), the mean squared error of the proposed estimator $t$, upto the first degree of approximation is obtained as 


$$
\begin{aligned}
& \operatorname{MSE}(t)=\left(\frac{1}{n}-\frac{1}{N}\right) \sum_{h=1}^{L} W_{h}\left[S_{y h}^{2}+R_{n}^{2} a_{h}^{2} S_{x h}^{2}+R_{m}^{2} c_{h}^{2} S_{z h}^{2}-2 R_{n} a_{h} S_{y x h}\right. \\
& \left.-2 R_{n} R_{m} a_{h} c_{h} S_{x z h}+2 c_{h} R_{m} S_{y z h}\right]
\end{aligned}
$$

where $R_{n}=\frac{\bar{Y}}{X_{n}}, R_{m}=\frac{\bar{Y}}{X_{m}}, X_{n}=\sum_{h=1}^{L} W_{h}\left(a_{h} \bar{X}_{h}+b_{h}\right)$ and $X_{m}=\sum_{h=1}^{L} W_{h}\left(c_{h} \bar{Z}_{h}+d_{h}\right)$.

Equation (2.4) can also be written as

$$
\operatorname{MSE}(t)=A+R_{n}^{2} B+R_{m}^{2} C-2 R_{n} D-2 R_{n} R_{m} E+2 R_{m} F
$$

where

$$
\begin{aligned}
& A=\left(\frac{1}{n}-\frac{1}{N}\right) \sum_{h=1}^{L} W_{h} S_{y h}^{2}, \\
& B=\left(\frac{1}{n}-\frac{1}{N}\right) \sum_{h=1}^{L} W_{h} a_{h}^{2} S_{x h}^{2}, \\
& C=\left(\frac{1}{n}-\frac{1}{N}\right) \sum_{h=1}^{L} W_{h} c_{h}^{2} S_{z h}^{2}, \\
& D=\left(\frac{1}{n}-\frac{1}{N}\right) \sum_{h=1}^{L} W_{h} a_{h} S_{y x h}, \\
& E=\left(\frac{1}{n}-\frac{1}{N}\right) \sum_{h=1}^{L} W_{h} a_{h} c_{h} S_{x z h} \text { and } \\
& F=\left(\frac{1}{n}-\frac{1}{N}\right) \sum_{h=1}^{L} W_{h} c_{h} S_{y z h} .
\end{aligned}
$$

The MSE of $t$ is minimum when

$$
\left\{\begin{array}{l}
R_{n}=\frac{D C-E F}{B C-E^{2}}=R_{n}^{*}(\text { say }) \\
R_{m}=\frac{D E-B F}{B C-E^{2}}=R_{m}^{*}(\text { say })
\end{array}\right.
$$

Putting (2.6) in (2.5), we get the minimum mean squared error of the proposed estimator $t$ as

$$
M S E_{\min }(t)=A[1-\rho]
$$

where $\rho=\frac{\left(C D^{2}+B F^{2}-2 D E F\right)}{A\left(B C-E^{2}\right)}$. 
Table 2.1: Some Known Members of $t$

Generated estimators $\quad$ Values of Constant

\begin{tabular}{lllll}
\hline \hline & $a_{h}$ & $b_{h}$ & $c_{h}$ & $d_{h}$ \\
\cline { 2 - 5 }$\hat{\bar{Y}}_{P S}^{R}=\bar{y}_{P S}$ & 0 & 1 & 0 & 1
\end{tabular}

Usual unbiased estimator

\begin{tabular}{lllll}
\hline$\hat{\bar{Y}}_{P S}^{R}=\bar{y}_{P S}\left(\frac{\bar{X}}{\bar{x}_{P S}}\right)$ & 1 & 0 & 0 & 1 \\
$\hat{\bar{Y}}_{P S}^{P}=\bar{y}_{P S}\left(\frac{\bar{z}_{P S}}{\bar{Z}}\right)$ & 0 & 1 & 1 & 0
\end{tabular}

Ige and Trapthi (1987) estimators

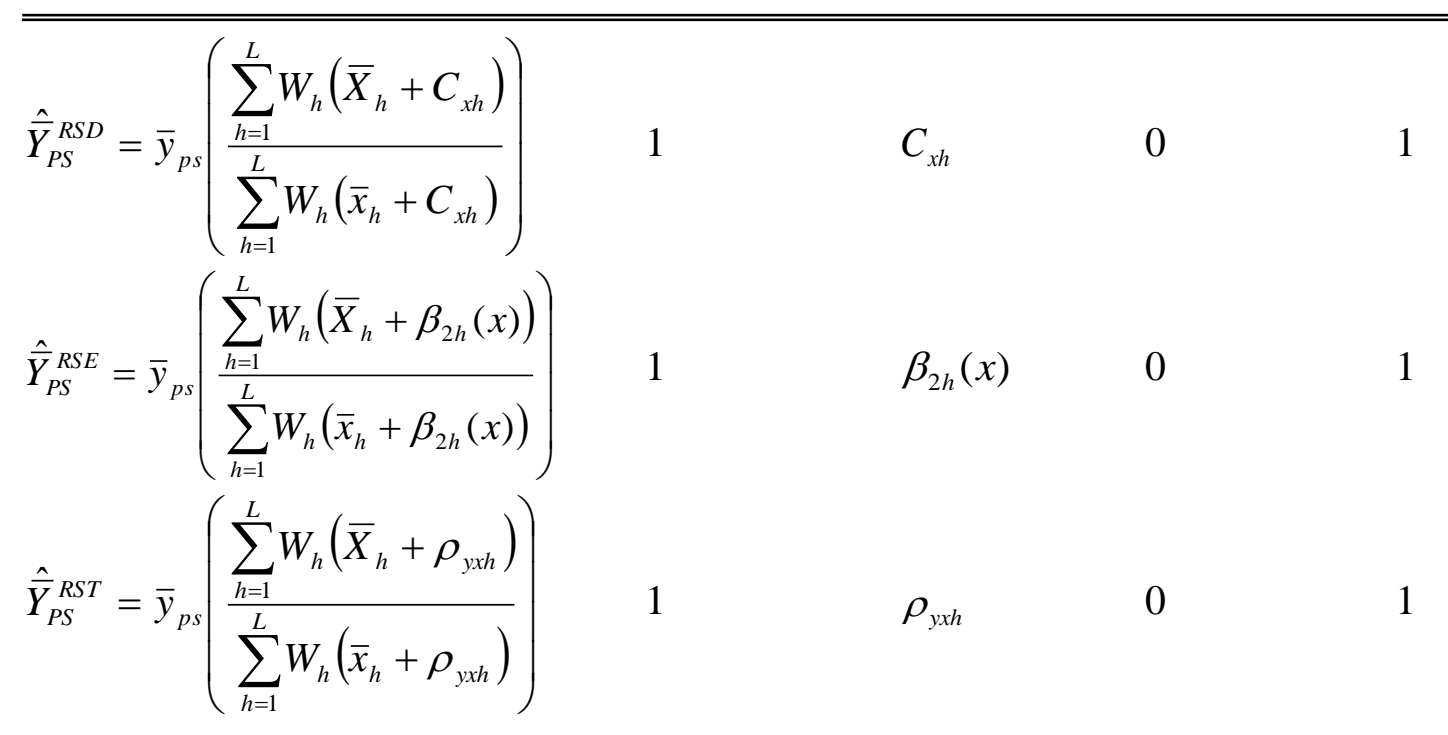

Chouhan (2012) estimators

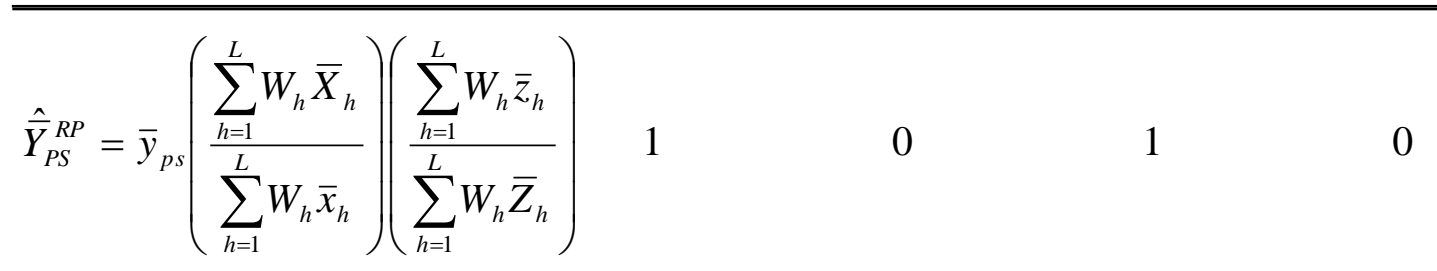

Tailor et al. (2016) estimator

$$
\begin{aligned}
t_{1}=\bar{y}_{p s}\left(\frac{\sum_{h=1}^{L} W_{h}\left(\bar{X}_{h}+C_{x h}\right)}{\sum_{h=1}^{L} W_{h}\left(\bar{x}_{h}+C_{x h}\right)}\right) & 1 \\
& \times\left(\frac{\sum_{h=1}^{L} W_{h}\left(\bar{z}_{h}+C_{z h}\right)}{\sum_{h=1}^{L} W_{h}\left(\bar{Z}_{h}+C_{z h}\right)}\right)
\end{aligned}
$$




$$
\begin{aligned}
& t_{2}=\bar{y}_{p s}\left(\frac{\sum_{h=1}^{L} W_{h}\left(\bar{X}_{h}+\beta_{2 h}(x)\right)}{\sum_{h=1}^{L} W_{h}\left(\bar{x}_{h}+\beta_{2 h}(x)\right)}\right) \quad 1 \quad \beta_{2 h}(x) \quad 1 \quad \beta_{2 h}(z) \\
& \times\left(\frac{\sum_{h=1}^{L} W_{h}\left(\bar{z}_{h}+\beta_{2 h}(z)\right)}{\sum_{h=1}^{L} W_{h}\left(\bar{Z}_{h}+\beta_{2 h}(z)\right)}\right) \\
& t_{3}=\bar{y}_{p s}\left(\frac{\sum_{h=1}^{L} W_{h}\left(\bar{X}_{h}+\rho_{y x h}\right)}{\sum_{h=1}^{L} W_{h}\left(\bar{x}_{h}+\rho_{y x h}\right)}\right) \\
& \times\left(\frac{\sum_{h=1}^{L} W_{h}\left(\bar{z}_{h}+\rho_{y z h}\right)}{\sum_{h=1}^{L} W_{h}\left(\bar{Z}_{h}+\rho_{y z h}\right)}\right)
\end{aligned}
$$

\section{Efficiency Comparisons}

From (1.2), (1.5), (1.6), (1.10), (1.11), (1.12), (1.16) (1.17), (1.18), (1.20) and (2.7), we conclude that the proposed class of ratio-cum- product type estimators $t$ would be more efficient than

(i) $\quad \bar{y}_{P S}$ if

$$
\rho>0
$$

(ii) $\quad \hat{\bar{Y}}_{P S}^{R}$ if

$$
\rho \sum_{h=1}^{L} W_{h} S_{y h}^{2} \sum_{h=1}^{L} W_{h}\left(R_{1}^{2} S_{x h}^{2}-2 R_{1} S_{y x h}\right)>0,
$$

(iii) $\quad \hat{\bar{Y}}_{P S}^{P}$ if

$$
\rho \sum_{h=1}^{L} W_{h} S_{y h}^{2}+\sum_{h=1}^{L} W_{h}\left(R_{1}^{2} S_{z h}^{2}+2 R_{2} S_{y z h}\right)>0,
$$


(iv) $\quad \hat{\bar{Y}}_{P S}^{R S D}$ if

$\rho \sum_{h=1}^{L} W_{h} S_{y h}^{2}+\sum_{h=1}^{L} W_{h}\left(R_{n 1}^{2} S_{x h}^{2}-2 R_{n 1} S_{y x h}\right)>0$,

(v) $\hat{\bar{Y}}_{P S}^{R S E}$ if

$\rho \sum_{h=1}^{L} W_{h} S_{y h}^{2}+\sum_{h=1}^{L} W_{h}\left(R_{n 2}^{2} S_{x h}^{2}-2 R_{n 2} S_{y x h}\right)>0$,

(vi) $\hat{\bar{Y}}_{P S}^{R S T}$ if

$\rho \sum_{h=1}^{L} W_{h} S_{y h}^{2}+\sum_{h=1}^{L} W_{h}\left(R_{n 3}^{2} S_{x h}^{2}-2 R_{n 3} S_{y x h}\right)>0$,

(vii) $\hat{\bar{Y}}_{P S}^{P S D}$ if

$\rho \sum_{h=1}^{L} W_{h} S_{y h}^{2}+\sum_{h=1}^{L} W_{h}\left(R_{m 1}^{2} S_{z h}^{2}+2 R_{m 1} S_{y z h}\right)>0$,

(viii) $\hat{\bar{Y}}_{P S}^{P S E}$ if

$\rho \sum_{h=1}^{L} W_{h} S_{y h}^{2}+\sum_{h=1}^{L} W_{h}\left(R_{m 2}^{2} S_{z h}^{2}+2 R_{m 2} S_{y z h}\right)>0$,

(ix) $\quad \hat{\bar{Y}}_{P S}^{P S T}$ if

$\rho \sum_{h=1}^{L} W_{h} S_{y h}^{2}+\sum_{h=1}^{L} W_{h}\left(R_{m 3}^{2} S_{z h}^{2}+2 R_{m 3} S_{y z h}\right)>0$,

(x) $\quad \hat{\bar{Y}}_{P S}^{R P}$ if

$\rho \sum_{h=1}^{L} W_{h} S_{y h}^{2}+\sum_{h=1}^{L} W_{h}\left[R_{1}^{2} S_{x h}^{2}+R_{2}^{2} S_{z h}^{2}-2\left(R_{1} S_{y x h}+R_{1} R_{2} S_{x z h}-R_{2} S_{y z h}\right)\right]>0$

Expressions (3.1) and (3.10) are the conditions under which the proposed class of ratiocum-product type estimator $t$ is better than usual unbiased estimator $\bar{y}_{P S}$, Ige and Tripathi (1989) estimators $\hat{\bar{Y}}_{P S}^{R}$ and $\hat{\bar{Y}}_{P S}^{P}$, Chouhan (2012) estimators $\hat{\bar{Y}}_{P S}^{R S D}, \hat{\bar{Y}}_{P S}^{R S E}$ and $\hat{\bar{Y}}_{P S}^{R S T}$, Tailor et al. (2016) estimator $\hat{\bar{Y}}_{P S}^{R P}$ and other considered estimators $\hat{\bar{Y}}_{P S}^{P S D}, \hat{\bar{Y}}_{P S}^{P S E}$ and $\hat{\bar{Y}}_{P S T}^{P S}$. 


\section{Study on the particular members of the proposed class of ratio-cum- product type estimators $t$}

To illustrate the general result, we have considered the new members $t_{1}, t_{2}$ and $t_{3}$ of the proposed class of ratio-cum- product type estimators $t$ defined in table 6.2.1 as

$$
\begin{aligned}
& t_{1}=\bar{y}_{p s}\left(\frac{\sum_{h=1}^{L} W_{h}\left(\bar{X}_{h}+C_{x h}\right)}{\sum_{h=1}^{L} W_{h}\left(\bar{x}_{h}+C_{x h}\right)}\right)\left(\frac{\sum_{h=1}^{L} W_{h}\left(\bar{z}_{h}+C_{z h}\right)}{\sum_{h=1}^{L} W_{h}\left(\bar{Z}_{h}+C_{z h}\right)}\right), \\
& t_{2}=\bar{y}_{p s}\left(\frac{\sum_{h=1}^{L} W_{h}\left(\bar{X}_{h}+\beta_{2 h}(x)\right)}{\sum_{h=1}^{L} W_{h}\left(\bar{x}_{h}+\beta_{2 h}(x)\right)}\right)\left(\frac{\sum_{h=1}^{L} W_{h}\left(\bar{z}_{h}+\beta_{2 h}(z)\right)}{\sum_{h=1}^{L} W_{h}\left(\bar{Z}_{h}+\beta_{2 h}(z)\right)}\right)
\end{aligned}
$$

and

$$
t_{3}=\bar{y}_{p s}\left(\frac{\sum_{h=1}^{L} W_{h}\left(\bar{X}_{h}+\rho_{y x h}\right)}{\sum_{h=1}^{L} W_{h}\left(\bar{x}_{h}+\rho_{y x h}\right)}\right)\left(\frac{\sum_{h=1}^{L} W_{h}\left(\bar{z}_{h}+\rho_{y z h}\right)}{\sum_{h=1}^{L} W_{h}\left(\bar{Z}_{h}+\rho_{y z h}\right)}\right)
$$

Upto the first degree of approximation, the mean squared errors of the estimators $t_{1}, t_{2}$ and $t_{3}$ are obtained as

$$
\begin{aligned}
& \operatorname{MSE}\left(t_{1}\right)=\left(\frac{1}{n}-\frac{1}{N}\right) \sum_{h=1}^{L} W_{h}\left[S_{y h}^{2}+R_{n 1}^{2} S_{x h}^{2}+R_{m 1}^{2} S_{z h}^{2}-2 R_{n 1} S_{y x h}-2 R_{n 1} R_{m 1} S_{x z h}+2 R_{m 1} S_{y z h}\right] \\
& \operatorname{MSE}\left(t_{2}\right)=\left(\frac{1}{n}-\frac{1}{N}\right) \sum_{h=1}^{L} W_{h}\left[S_{y h}^{2}+R_{n 2}^{2} S_{x h}^{2}+R_{m 2}^{2} S_{z h}^{2}-2 R_{n 2} S_{y x h}-2 R_{n 2} R_{m 2} S_{x z h}+2 R_{m 2} S_{y z h}\right]
\end{aligned}
$$

and

$$
\operatorname{MSE}\left(t_{3}\right)=\left(\frac{1}{n}-\frac{1}{N}\right) \sum_{h=1}^{L} W_{h}\left[S_{y h}^{2}+R_{n 3}^{2} S_{x h}^{2}+R_{m 3}^{2} S_{z h}^{2}-2 R_{n 3} S_{y x h}-2 R_{n 3} R_{m 3} S_{x z h}+2 R_{m 3} S_{y z h}\right]
$$

\section{Empirical Study}

To exhibit the performance of the proposed estimators, two population data sets are being considered. Descriptions of data sets are given by

\section{Table 5.1: Population I- [Source: Chouhan (2012)]}

$y:$ Productivity (MT/Hectare)

$x$ : Production in ' 000 Tons and 
$z$ : Area in '000 Hectare

\begin{tabular}{lll}
\hline \hline Constant & Stratum I & Stratum II \\
\hline \hline$N_{h}$ & 10 & 10 \\
$n_{h}$ & 4 & 4 \\
$\bar{Y}_{h}$ & 1.70 & 3.67 \\
$\bar{X}_{h}$ & 10.41 & 289.14 \\
$\bar{Z}_{h}$ & 6.32 & 80.67 \\
$S_{y h}$ & 0.50 & 1.41 \\
$S_{x h}$ & 3.53 & 111.61 \\
$S_{z h}$ & 1.19 & 10.82 \\
$S_{y x h}$ & 1.60 & 144.87 \\
$S_{y z h}$ & -0.05 & -7.04 \\
$S_{x z h}$ & 1.38 & -92.02 \\
$\beta_{2 h}(x)$ & 1.97 & 2.90 \\
$\beta_{2 h}(z)$ & 4.12 & 3.66 \\
\hline \hline
\end{tabular}

Table 5.2: Population II- [Source: Murthy (1967), p 228]

$z:$ Number of workers

$y:$ Output and

$x$ : Fixed capital

\begin{tabular}{lll}
\hline \hline Constant & Stratum I & Stratum II \\
\hline \hline$N_{h}$ & 5 & 5 \\
$n_{h}$ & 2 & 2 \\
$\bar{Y}_{h}$ & 1925.8 & 315.6 \\
$\bar{X}_{h}$ & 214.4 & 333.8 \\
$\bar{Z}_{h}$ & 51.80 & 60.60 \\
$S_{y h}$ & 615.92 & 340.38 \\
$S_{x h}$ & 74.87 & 66.35 \\
$S_{z h}$ & 0.75 & 4.84 \\
$S_{y x h}$ & 39360.68 & 22356.50 \\
$S_{y z h}$ & 411.16 & 1536.24 \\
$S_{x z h}$ & 38.08 & 287.92 \\
$\beta_{2 h}(x)$ & 1.88 & 2.32 \\
$\beta_{2 h}(z)$ & 1.84 & 1.49 \\
\hline \hline
\end{tabular}


Table 5.3: Percent Relative Efficiencies of the estimators $\bar{y}_{P S}, \hat{\bar{Y}}_{P S}^{R}, \hat{\bar{Y}}_{P S}^{P}, \hat{\bar{Y}}_{P S}^{R S D}, \hat{\bar{Y}}_{P S}^{R S E}$, $\hat{\bar{Y}}_{P S}^{R S T}, \hat{\bar{Y}}_{P S}^{P S D}, \hat{\bar{Y}}_{P S}^{P S E}, \hat{\bar{Y}}_{P S}^{P S T}, \hat{\bar{Y}}_{P S}^{R P}, t_{1}, t_{2}$ and $t_{3}$ with respect to $\bar{y}_{P S}$.

\begin{tabular}{lll} 
Estimators & \multicolumn{2}{l}{ Percent Relative Efficiencies } \\
\cline { 2 - 3 } & Population I & Population II \\
\hline \hline $\bar{y}_{P S}$ & 100.00 & 100.00 \\
$\hat{\bar{Y}}_{P S}^{R}$ & 223.74 & 313.75 \\
$\hat{\bar{Y}}_{P S}^{P}$ & 123.31 & 85.02 \\
$\hat{\bar{Y}}_{P S}^{R S D}$ & 225.20 & 376.69 \\
$\hat{\bar{Y}}_{P S}^{R S}$ & 233.80 & 384.14 \\
$\hat{\bar{Y}}_{P S}^{R S T}$ & 227.49 & 378.73 \\
$\hat{\bar{Y}}_{P S}^{P S D}$ & 123.36 & 85.03 \\
$\hat{\bar{Y}}_{P S}^{P S E}$ & 123.38 & 85.43 \\
$\hat{\bar{Y}}_{P S T}^{P S}$ & 123.47 & 85.25 \\
$\hat{\bar{Y}}_{P S}^{R P}$ & 288.16 & 258.07 \\
$t_{1}$ & $\mathbf{2 9 1 . 0 7}$ & $\mathbf{4 0 4 . 8 5}$ \\
$t_{2}$ & $\mathbf{3 1 2 . 9 1}$ & $\mathbf{4 0 9 . 5 4}$ \\
$t_{3}$ & $\mathbf{2 9 4 . 3 1}$ & $\mathbf{4 0 5 . 4 2}$ \\
\hline \hline & & \\
\hline \hline
\end{tabular}

\section{Conclusion}

A class of ratio-cum-product type estimators for population mean has been defined. The usual unbiased estimator, Ige and Tripathi (1989) estimators, Chouhan (2012) estimators and Tailor et al. (2016) estimator have been identified to be the member of the proposed class of ratio-cum-product type estimators $t$. Section 3 provides the conditions under which the proposed estimator $t$ has less mean squared error as compared to the mean squared error of the other considered estimators. It is observed that particular members $t_{1}, t_{2}$ and $t_{3}$ have higher percent relative efficiencies in comparison to usual unbiased estimator $\bar{y}_{P S}$, Ige and Tripathi (1989) estimators $\hat{\bar{Y}}_{P S}^{R}$ and $\hat{\bar{Y}}_{P S}^{P}$, Chouhan (2012) estimators $\hat{\bar{Y}}_{P S}^{R S D}, \hat{\bar{Y}}_{P S}^{R S E}$ and $\hat{\bar{Y}}_{P S}^{R S T}$, Tailor et al. (2016) estimator $\hat{\bar{Y}}_{P S}^{R P}$ and other estimators $\hat{\bar{Y}}_{P S}^{P S D}, \hat{\bar{Y}}_{P S}^{P S E}$ and $\hat{\bar{Y}}_{P S}^{P S T}$. Hence, it can be concluded that the proposed members $t_{1}, t_{2}$ and $t_{3}$ are recommended for use in practice. Thus, it has been concluded that there is an enough scope of generating better estimators from the proposed class of ratio-cumproduct type estimator $t$ using suitable known parameters of auxiliary variates. 


\section{Acknowledgment}

The authors are grateful to the reviewers for their constructive comments and valuable suggestions regarding improvement of the article.

\section{References}

1. Agrawal, M. C. and Pandey, K. B. (1993). An efficient estimator in poststratification. Metron 51, 179-188.

2. Chouhan, S. (2012). Improved estimation of parameters using auxiliary information in sample surveys. Ph.D. Thesis, Vikram University, Ujjain, M.P., India.

3. Cochran, W. G. (1940). The estimation of the yields of the cereal experiments by sampling for the ratio of grain to total produce. J. Agril. Soc., 30, 262-275.

4. Holt, D. and Smith, T. M. F. (1979). Post-stratification. J. Roy. Statist. Soc., 142, A, 33-46.

5. Ige, A. F. and Tripathi, T. P. (1989). Estimation of population mean using poststratification and auxiliary information. Abacus, 18, 2, 265-276

6. Jagers, P. (1968). Post stratification against bias in sampling. Int. Statist. Rev., 55, 159-167.

7. Jagers, P., Oden, A. and Trulsson, L. (1985). Post stratification and ratio estimation: Usages of auxiliary information in surveys sampling and opinion polls. Int. Statist. Rev., 53, 221-238

8. Johnston, J. (1972). Econometric Methods, 2nd ed., Tokyo: McGraw-Hill.

9. Lone H. A. and Tailor R. (2015). Dual to Separate Product Type Exponential Estimator in Sample Surveys. J. Statist. Appl. Prob. Lett. 2, 1, 89-96

10. Lone, H. A. and Tailor, R. (2014). Dual to Separate Ratio Type Exponential Estimator in Post-Stratification. J. Statist. Appl. Prob. 3, 3, 425-432

11. Murthy, M. N. (1967). Sampling Theory and Methods, Statistical Publishing Society. Calcutta, India.

12. Robson, D. S. (1957). Application of multivariate polykays to the theory of unbiased ratio-type estimation. J. Amer. Statist. Assoc., 52, 511-522.

13. Singh, H. P. and Ruiz Espejo, M. (2003). On Linear regression and ratio-product estimation of a finite population mean. The Statistician, 52, 1, 59-67.

14. Singh, M. P. (1967). Ratio-cum-product method of estimation. Metrika, 12, 1, 3443.

15. Stephan, F. (1945). The expected value and variance of the reciprocal and other negative powers of a positive Bernoullian variate. Ann. Math. Statist. 16, 50-61.

16. Tailor, R., Jatwa, S. K. and Lone, H. A. (2015). Dual to ratio and product type estimators in case of post-stratification. J. Mod. Appl. Statist. Meth. ( in press)

17. Tailor, R., Lone, H. A., Pandey, R., and Kumar, M. (2016). Ratio-cum-product type estimator in case of post- stratification. Math. Sci. Lett. 5, 1, 103-106.

18. Tailor, R., Parmar, R., Kim, J. M. and Tailor, R. (2011). Ratio-cum-product estimators of population mean using known population parameters. Comm. Kor. Statist. Soc. 18, 2, 155-164. 